Background The large number of sexually transmitted diseases cases caused globally each year by Chlamydia trachomatis has made this organism a World Health Organization priority for vaccine development. Even after a decade of availability of C. trachomatis genome sequence, no promising vaccine has seen the light of the day. This clearly indicates the challenges in discovering new vaccines against this organism but also suggests a gap in our current understanding of Chalmydial biology. We attempt to bridge this gap by carrying out extensive annotation of hypothetical proteins of $\mathrm{C}$. trachomatis and further identification of candidate genes that might be involved during the immune response against this organism. In this study, we have shortlisted proteins secreted by the general export pathway of C. trachomatis from among the hypothetical proteins of this organism with an aim to identify novel vaccine candidate gene/s.

Methodology Characterization of the proteins was carried out using various Bioinformatic tools, Pfam, Tigrfam, Scanprosite, CDD, Signal P, SigPred, TMPred, TMHMM and Lipo P.

Results 336 hypothetical proteins were deduced from the C. trachomatis genome and were analysed with several software tools for functional annotation. Next we identified the hypothertical proteins are secreted via the general export pathway (GEP) using bioinformatics approach. We were able to classify the shortlisted proteins into three broad categories as outer membrane proteins, secretory proteins and lipoproteins. These shortlisted candidate proteins could possibly induce protective immunity or elicit immune responses of diagnostic value. Few of them can further turn out to be good vaccine candidate genes as well.

Conclusion The identification of novel secreted proteins of C. trachomatis opens the way to studies on their subcellular localization and to the immunological characterization of these proteins to define their potential for immunological diagnosis and/or vaccine design.

\section{P5.106 CHARTING THE PATH FOR HUMAN PAPILLOMAVIRUS (HPV) VACCINE INTRODUCTION IN KENYA: ASSESSING HPV VACCINE ACCEPTABILITY AMONG CAREGIVERS AND OPINION LEADERS IN NYANZA PROVINCE, KENYA}

doi:10.1136/sextrans-2013-051184.1150

'A L Friedman, 'E Dunne, ${ }^{2} \mathrm{~K}$ Onyango, 'M Habel, 'J Ford, ' J Kinsey, 'L Markowitz, ${ }^{3} \mathrm{P}$ Phillips-Howard, ${ }^{2 K}$ Laserson. 'US Centers for Disease Control \& Prevention, Atlanta, GA, United States; ${ }^{2}$ KEMRI/CDC, Kisumu, Kenya; ${ }^{3}$ iverpool School of Tropical Medicine, Liverpool, UK

Background Cervical cancer is the second most common cancer diagnosed, and the leading cause of cancer-related mortality among women in Kenya. Kenya's Ministry of Health has outlined new prevention strategies, including support for vaccination. Formative research is critical to identify information, mobilisation and communication needs for vaccine introduction. To inform vaccine mobilisation and communication efforts in Kenya, this study sought to explore HPV vaccine-related community knowledge, attitudes, beliefs, and acceptability.

Methods We conducted five focus groups (FG) among caregivers of girls aged $9-12$ years $(n=56)$, and 12 interviews with community opinion leaders in four locations of Nyanza Province, Kenya. Information was collected about participant knowledge, attitudes and beliefs regarding vaccines, cancer, cervical cancer and HPV; and perceived benefits/barriers to HPV vaccination. FG transcripts and interview notes were reviewed and analysed by a team of four researchers using thematic content analysis. FG data were analysed using NVivo8, and concept matrices were used for interview notes. Results Awareness of vaccines and cancer was high among caregivers and opinion leaders, but low for cervical cancer, HPV and HPV vaccines. Whereas cancer was feared as a fatal disease, vaccines were generally well accepted. Once informed, participants were eager to support HPV vaccination for their daughters/communities. Protection against cervical cancer was perceived as a key benefit. Potential barriers to vaccination included religious/cultural beliefs; confusion/suspicion about intended vaccination effort; and concerns about vaccine safety and side effects (infertility). Most believed these barriers could be overcome with effective community mobilisation/education.

Conclusion The success of an HPV vaccination programme will depend critically on information, communication and social mobilisation - not only for raising awareness of cervical cancer and the need for vaccination, but for preventing possible misconceptions and rumours from arising. Recommendations are made to inform Kenya's communication and mobilisation strategies in preparation for vaccine introduction.

\section{P5.107 CLINICAL PRACTICE GUIDELINE FOR SEXUALLY TRANSMITTED INFECTIONS AND OTHER INFECTION OF THE GENITAL TRACT - SYNDROMIC MANAGEMENT - 2012}

doi:10.1136/sextrans-2013-051184.1151

${ }^{1}$ H Gaitan Duarte, ${ }^{2} \mathrm{~A}$ Rodriguez, ${ }^{3} \mathrm{E}$ Angel-Muller, ${ }^{4} \mathrm{H}$ Lopez-Gomez, ${ }^{5} \mathrm{~S}$ Estrada, ${ }^{6}$ CINETS A. 'National University of Colombia, Bogota, Colombia; ${ }^{2}$ Clinical Research Institute, National University of Colombia, Bogota, Colombia; ${ }^{3}$ Obstetrics and Gynecology Departament, National University of Colombia, Bogota, Colombia; ${ }^{4}$ Colombian Society of Urology, Bogota, Colombia; ${ }^{5}$ Colombian Association of Infectology, Bogota, Colombia; ${ }^{6}$ Universidad Nacional de Colombia, Universidad Javeriana, Universidad de Antioquia, Bogota, Colombia

Objective To develop a Clinical Practice Guidelines for the Syndromic management of Sexual Transmitted Infections and other Genital Tract Infections in patients between 14 and 75 years old using the current international standards.

Methods The Guidelines developer group (GDG) was composed taking into account a multidisciplinary team as well as user/ patients' point of view. The scope, questions and relevant outcomes were first defined and then a search of available CPGs was done looking for international and regional repositories and also in Medline and LILACS. The CPGs that accomplish the inclusion exclusion criteria were assessed using the AGREE II instrument. None of the evaluated guidelines were suitable to be adapted to the Colombian requirements. Based on this fact, a search for Systematic Reviews, RCT and observational studies was initiated in order to develop the novo CPG. Evidence tables were built and recommendations were elaborated following the GRADE approach using informal and formal consensus.

Results A new GPC for the management of the patients with cervicitis, urethritis, genital ulcer disease, pelvic inflammatory disease and vaginal discharge is presented. The CPG includes 80 recommendations for the diagnosis and treatment of the index patient and its partner and also for the pregnant women with GTI. The implementation plan is based on the administration of a single dose for each of the causal germens in each syndrome.

Conclusion A new evidence-based guideline is presented for Syndromic management of Sexual Transmitted Infections and other Genital Tract Infections which could be suitable for adaptation in settings of constrained resources. Source of funds: Ministry of Health of Colombia and COLCIENCIAS.

\section{P5.108 ATHEROSCLEROTIC CAROTID DISEASE AND CARDIOVASCULAR RISK IN HIV-INFECTED PATIENTS}

doi:10.1136/sextrans-2013-051184.1152

J C Hueb, P Salmazo, A N Barbosa, R Terribilli, B Matsubara. Faculdade de Medicina de Botucatu, Botucatu, Brazil

Introduction: Although survival has been improved in HIVinfected patients, the risk for atherosclerotic diseases has increased. 
Objective To evaluate the effect of HIV infection on atherosclerosis in asymptomatic patients.

Subjects and methods Study design: observational, prospective case-control study, including 124 consecutive male and female HIVinfected patients, older than 18 year (HIV-group). Results were compared with 130 healthy volunteers of same gender and age (Control-group).

Study protocol: Clinical evaluation followed by ultrasound exam of carotids for carotid intimal-medial thickness (CIMT) measurement. The presence of atherosclerosis plaques was recorded.

Statistical analysis: Chi-square test and linear regression analysis. Significance level: $p<0.05$.

Result Age (HIV-group:43.87 \pm 11.31 vs Control-group: $42.9 \pm$ $11.54, p=0.324)$ was similar in both groups. There was $52.00 \%$ male in HIV-group and $54.00 \%$ in Control group. Atherosclerotic plaque was identified in $30.64 \%$ of the patients HIV and in $3.84 \%$ of the control ( $p<0.001$ ). Surprisingly, $22 \%$ of HIV-infected patients, classified at low risk score Freminghan, presented plaques in the carotid arteries.

For controls, there was an increase of $0.068 \mathrm{~mm}$ in the CIMT for each one-year increase in age (OR: 1.068; CI95\%: 1.03-1.107; $\mathrm{p}<0.001)$. The presence of HIV increased this increment for tenfold (OR: 10.7; CI95\%: 3.58-31.76; $\mathrm{p}<0.001$ ). There was an interaction between age and HIV-infection to increase CIMT ( $p<0.001)$.

Conclusions Our results indicated that: 1 - patients with HIV are at higher risk for atherosclerosis in the carotid artery than control individuals. 2- The effect of age on risk for atherosclerosis occurs in both groups, however it is more remarkable in HIV-infected patients.

\section{P5.109 KNOWLEDGE AND UPTAKE OF HIV INTERVENTION AND PREVENTION SERVICES IN MANICALAND, ZIMBABWE: CURRENT DETERMINANTS OF COVERAGE AND PROGRESS OVER TIME}

doi:10.1136/sextrans-2013-051184.1153

'N Schur, ${ }^{1,2} \mathrm{C}$ Nyamukapa, 1,2S Gregson. 'Department of Infectious Disease Epidemiology, Imperial College London, London, UK; ${ }^{2}$ Biomedical Research and Training Institute, Harare, Zimbabwe

Background Zimbabwe has experienced one of the world's most severe HIV crises since the first reported case of AIDS in the country in 1985. Over the last decade, the Zimbabwe Government has implemented a range of services to prevent new infections and to support people living with HIV, e.g. HIV testing and counselling (HTC), anti-retroviral treatment (ART), prevention of mother-tochild transmission (PMTCT), and male circumcision (MC).

We describe trends and patterns in knowledge and uptake of these services, investigate individual-level factors associated with greater levels of coverage, and analyse shortfalls in order to inform future policies on the scale-up of HIV services.

Methods Data from the latest round of a large $(n \approx 12,000)$ population-based cohort study conducted between 2009 and 2011 in eastern Zimbabwe will be used in logistic regression models to analyse associations between demographic, socio-economic, behavioural and other factors, and knowledge and uptake of services. Temporal trends in uptake of services will be measured using data from earlier rounds of the study.

Results HIV prevalence in the general population of Manicaland province remained comparably high $(15.1 \%$; $95 \%$ confidence interval: $14.5 \%-15.7 \%)$. Preliminary univariate results suggest that knowledge of HIV services was high for HTC (82.3\%; 91.6\%-82.9\%) and PMTCT $(93.0 \% ; 92.0 \%-93.9 \%)$. Knowledge of ART was low overall (40.1\%; 39.3\%-40.9\%) but high in self-reported HIV-positives $(88.9 \% ; 86.8 \%-90.7 \%)$. Overall uptake of services in the last three years was moderate for HTC (44.0\%; 43.2\%-44.8\%) and PMTCT
(65.2\%; $57.1 \%-72.6 \%)$. Current uptake of ART in self-reported HIV positives (79.2\%; 76.3\%-81.8\%) almost reached universal coverage, which is defined at $85 \%$ in Zimbabwe, but only about every second lab-confirmed HIV positive person in the study population was on treatment $(52.2 \% ; 49.2 \%-55.1 \%)$. MC uptake in the male population remained low $(8.2 \% ; 7.5 \%-8.9 \%)$. Knowledge and uptake of services varied by sex and age and may also differ by other factors.

\section{P5.110 MULTIPROFESSIONAL APPROACH FOR THE DEVELOPMENT OF THE NATIONAL STIS MANAGEMENT GUIDELINES IN LITHUANIA}

doi:10.1136/sextrans-2013-051184.1154

'V Kucinskiene, 'S Valiukeviciene, 'A Vitkauskiene, ${ }^{2} \mathrm{M}$ Domeika, Eastern European Network for SexualReproductive Health. 'Department of Skin and Venereal Diseases, Medical Academy, Lithuanian University of Health Sciences, Kaunas, Lithuania; ${ }^{2}$ Department of Control and Prevention of Communicable Diseases Uppsala County Council, Uppsala, Sweden

Background During many decades in the majority of Eastern European Countries, persons with lower genital tract infections were treated exceptionally by the veneologist. Today dermatovenereologist sees less than $40 \%$ of these patients. The rest of the patients is treated and managed by gynaecologists, general physicians, urologists etc. Therefore in developing STI patient management approaches a multiprofessional approach is crucial.

Methods Recently, collaborating effort to prepare STI patient management guidelines in Lithuania was undertaken. Dermatovenereologists, gynaecologists, urologists, microbiologists, general practitioners and other STI patients managing specialists for the first time gathered together. IUSTI, WHO, CDC and EE SRHR guidelines were used.

Results Collaborating project resulted in development of evidencebased STI patient management guidelines, containing the information about the: (i) who should be tested; (ii) what methods are to be used; (iii) recommended treatment regiments; (iv) partner management strategies; (v) follow up approaches; (vi) case reporting and (vi) counselling. Syndromic approach for the management of urethritis and cervicitis as well as complications has also been described. Over 1200 copies of the guidelines have been disseminated to medical professionals and made available for medical students. The electronic version of the document has been presented at the website www. kaunoklinikos. It and can be accessed to any specialist and medical student with no charge. During the preparation of the guidelines the main discrepancies with the international practise identified and eliminated, as e.g. use of serology for diagnosis of chlamydial infection, the use microscopy and not culture, no NAATS for the diagnosis of gonorrhoea, treatment of early syphilis by short acting penicillin, preventive treatment of syphilis for pregnant women, etc. Conclusion STI management guidelines became an excellent example of the multiprofessional collaboration in Lithuania, bringing international evidence-based approaches across the professional barriers of all medical professions, involved in management of STIpatients.

\section{P.06 - Health Policy and Programme Sciences Track}

P6.001 FACING THE FUTURE TOGETHER: EMBODYING
APPRECIATIVE INQUIRY AND STAFF ENGAGEMENT IN
PLANNING CHANGE IN SEXUAL HEALTH SERVICES IN
GLASGOW, SCOTLAND, UK

doi:10.1136/sextrans-2013-051184.1155

${ }^{1,2} \mathbf{R}$ llett. 'Sandyford Sexual Health Services, Glasgow, UK; ${ }^{2}$ University of Glasgow, Glasgow, UK 